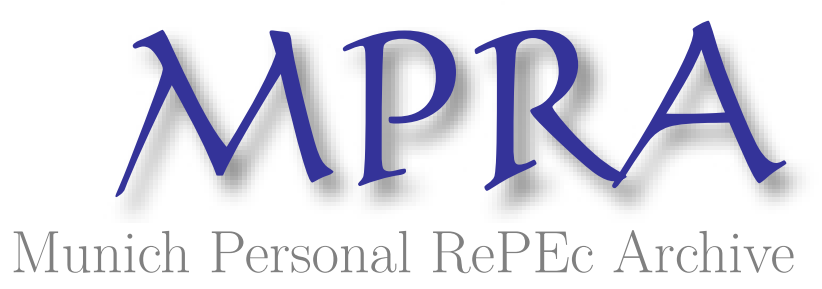

\title{
Games of capacity allocation in many-to-one matching with an aftermarket
}

Mumcu, Ayse and Saglam, Ismail

Bogazici University, TOBB-ETU

19 June 2008

Online at https://mpra.ub.uni-muenchen.de/9239/

MPRA Paper No. 9239, posted 20 Jun 2008 00:33 UTC 


\title{
Games of Capacity Allocation in Many-to-One Matching with an Aftermarket*
}

\author{
Ayşe Mumcu \\ Department of Economics, Bogazici University
}

AND

ISMAIL SAGLAM ${ }^{\dagger}$

Department of Economics, TOBB University of Economics and Technology

In this paper, we study many-to-one matching (hospital-intern markets) with an aftermarket. We analyze the Nash equilibria of capacity allocation games, in which preferences of hospitals and interns are common knowledge and every hospital determines a quota for the regular market given its total capacity for the two matching periods. Under the intern-optimal stable matching system, we show that a pure-strategy Nash equilibrium may not exist. Common preferences for hospitals ensure the existence of equilibrium in weakly dominant strategies whereas unlike in games of capacity manipulation strong monotonicity of population is not a sufficient restriction on preferences to avoid the nonexistence problem. Besides, in games of capacity allocation, it is not true either that every hospital weakly prefers a mixed-strategy Nash equilibrium to any larger regular market quota profiles.

Keywords: Many-to-one matching; hospital-intern market; aftermarket; capacity allocation game

JEL Classification Numbers: C72; C78; D71

*The second author acknowledges the support of Turkish Academy of Sciences, in the framework of Distinguished Young Scientist Award Program (TUBA-GEBIP). The usual disclaimer applies.

${ }^{\dagger}$ Corresponding author. Department of Economics, TOBB University of Economics and Technology, Sogutozu Cad. 43, Sogutozu 06560, Ankara, Turkey. E-mail: ismail.saglam@etu.edu.tr 


\section{Introduction}

Over a decade, many markets ranging from entry-level labor markets (e.g., markets for medical fellows, gastroenterologists, clinical psychologists, law clerks) to school choice system and kidney exchange, have been redesigned drawing on the insights from the two-sided matching theory. One of the major concerns in design is the manipulability of the outcomes by either side that may arise in terms of preference or capacity manipulation. While preference manipulation refers to an agent's (from either side) effort to improve his or her match by misrepresenting his or her preferences, the capacity manipulation occurs in a many-to-one matching setting when a school/firm/hospital underreports its true capacity with the same purpose.

The existing studies on capacity manipulation consider incentives to manipulate in single-shot matching markets (Sönmez, 1997; Kojima, 2006; Konishi and Ünver, 2006). However, many markets, centralized or decentralized, convene to clear the unfilled capacities in an aftermarket. For instance, in college admissions, many colleges offer early and regular admissions programs. A scramble market for those who are not matched in the regular market has recently been designed for new Ph.D.s in Economics (www.aeaweb.org/joe/scramble). Also, National Residency Matching Program (NRMP) employs a similar scramble market in hospital-intern market (www.nrmp.org). In Turkey, secondary school placement mechanism centrally places students to unassigned seats in an aftermarket.

Theoretically, intertemporal capacity allocation, just like total capacity announcement, is a strategic decision in two-sided matching markets. In college admissions, many colleges have used in the last fifty years early admissions programs to improve the quality of the student intake. However, in some other cases, like in the NRMP, the direct ${ }^{1}$ use of intertemporal capacity allocation is prevented by disallowing hospitals to change their announced quota after the matching procedure has started. ${ }^{2}$

\footnotetext{
${ }^{1}$ We will discuss, in Conclusions, how quota reversions in the NRMP can be used as an indirect strategic device of intertemporal capacity allocation in hospital-intern markets.

${ }^{2}$ In the NRMP, each program sets a quota when participating in the match. If any change is made to the quota, the institutional official must approve the change before it
} 
Capacity manipulation in single-shot and two-sided matching markets has been well understood. Sönmez (1997) shows that in a single-period hospital-intern market there is no stable matching rule that is nonmanipulable by hospitals via underreporting capacities. Manipulation of capacities in hospital-intern markets is further analysed by Konishi and Ünver (2006), using a one-period game between hospitals. Under two most widely used matching rules, namely hospital-optimal and intern-optimal stable matching rules, Konishi and Ünver (2006) show that there may not be a pure-strategy equilibrium in general, and whenever a pure-strategy equilibrium exists, every hospital weakly prefers this equilibrium outcome to the outcome of any larger capacity profile. Konishi and Ünver (2006) consider two restrictions on preferences, each of which guarantees the existence of a pure-strategy equilibrium. The first restriction requires hospitals to always prefer a larger set of acceptable interns to a smaller set. By that, reporting the number of assigned interns is an equilibrium strategy if the matching rule is hospitaloptimal, whereas under the intern-optimal matching rule reporting the actual capacity is a weakly dominant strategy. The second restriction requires common preferences of one group of agents (hospitals or interns) over the other group and ensures that reporting the true capacity is always a weakly dominant strategy for hospitals. Kojima (2006) further investigates the game studied by Konishi and Ünver (2006) and establishes that there always exists a mixed-strategy equilibrium and every hospital weakly prefers a Nash equilibrium (pure or mixed) to any "larger" capacity profiles.

All of these previous studies point to the incentives for hospitals in underreporting capacities in single-shot hospital-intern markets. An immediate observation is the possibility of filling the unused capacities in the aftermarket via a centralized clearinghouse. ${ }^{3}$

can be used in the final match process. All change approvals must be made by the rank order list deadline. See the NRMP User Guide (2007).

${ }^{3}$ In some markets, the aftermarket is a decentralized market as the scramble market in the NRMP and the job market for new Ph.D.s in Economics. However, there are also cases where the aftermarket is operated via a centralized clearinghouse, like in secondary school placement in Turkey. The quota reversions in the NRMP mechanism also mimick a two-stage centralized market structure since the quota reversions are done within the matching process, right after the first round of the match. 
In this paper, we study capacity allocation in a two-period many-to-one matching market, involving a regular market and an aftermarket. There are two finite and disjoint sets of agents, say hospitals and interns. Each hospital has a finite overall capacity that limits the number of interns it can employ in the two periods, and each intern can be employed by at most one hospital during the whole matching process. Each hospital has a preference relation over the power set of interns that is responsive to its preference over the set of interns, and each intern has a preference relation over the set of hospitals and being unmatched. The capacities of hospitals together with the preference profiles of hospitals and interns constitute a matching environment.

In the regular decision period, each hospital announces out of its total capacity a regular market quota which it aims to fill according to its preference ordering. The regular market quotas of hospitals together with the preferences of hospitals and interns constitute a regular market.

The capacities of hospitals, the preferences of hospitals and interns, and the matchings achieved in the regular decision period altogether define an aftermarket.

An allocation in the regular decision period is a many-to-one regular market matching where no hospital is assigned more interns than its regular market quota and no intern is assigned more than one hospital. Given a binding regular market matching, an allocation in the aftermarket is a manyto-one aftermarket matching where all the assignments realized in the regular market are preserved, no hospital is assigned more interns than its overall capacity and no intern is assigned more than one hospital. We call any assignment that did not exist in the regular decision period and realize in the aftermarket as an aftermarket assignment. We assume that any intern rejected from a hospital in the regular market can reapply to that hospital in the aftermarket.

A matching in the regular market is stable if no intern prefers being unemployed to his or her assignment, no hospital prefers having an intern position vacant rather than filling it with one of its assignments, and there exists no unmatched hospital-intern pair such that the hospital prefers the intern to one of its assignments or keeping a vacant position and the intern prefers the hospital to his or her assignment. Given a matching realized in 
the regular decision period, a matching in the aftermarket period is stable if no intern having an aftermarket assignment prefers being unemployed to his or her assignment, no hospital prefers aftermarket intern position vacant rather than filling it with one of its aftermarket assignments, and there exists no unmatched hospital-intern pair such that the hospital prefers the intern to one of its aftermarket assignments or keeping a vacant position and the intern prefers the hospital to his or her aftermarket assignment.

A regular market matching rule selects a matching for every regular market, and is stable if it selects a stable matching for every regular market. An aftermarket matching rule selects a matching for every aftermarket. An aftermarket matching rule is stable at an aftermarket if it selects a stable matching for the given aftermarket.

A regular market rule and an aftermarket rule as an ordered pair form a matching system. A matching system is stable if it involves a stable regular market rule and an aftermarket matching rule that is stable at the aftermarket associated with the regular matching outcome of the regular market rule.

After the description of the basic structures of our two-period matching model, we define a game in which the total capacities of hospitals as well as the preferences of hospitals and interns are common knowledge and each hospital determines a quota for the regular decision period given its total capacity for the two periods. Under the intern-optimal stable matching system, we show that there may not be a pure-strategy Nash equilibrium, extending the result by Konishi and Ünver (2006), obtained in games of capacity manipulation. However, the capacity lemma of Konishi and Ünver (2006), establishing that any hospital's underreport of its capacity has a monotonic non-negative (non-positive) effect on the welfare of other hospitals (interns) does not hold in games of capacity allocation. The mixed-strategy equilibrium trivially exists in games of capacity allocation, while Kojima's (2006) generalization - that every hospital weakly prefers a non-degenerate mixedstrategy Nash equilibrium to any larger capacity profiles in games of capacity manipulation - fails to be true in games of capacity allocation. We also show that common preferences for hospitals ensure the existence of equilibrium in weakly dominant strategies. But, unlike in capacity manipulation games, 
strong monotonicity of population is not a sufficient restriction on preferences to avoid the nonexistence problem.

The organization of the rest of the paper is as follows: Section 2 introduces the basic structures and a game of capacity allocation. Section 3 presents our results. Finally, Section 4 concludes.

\section{Model}

\subsection{Basic Structures}

\subsubsection{Matching Environment}

A matching environment is denoted by the list $\left(H, I, q_{H}, R\right)$. The first two components are non-empty, finite and disjoint sets of hospitals $H=\left\{h_{1}, h_{2}, \ldots\right.$, $\left.h_{m}\right\}$ and interns $I=\left\{i_{1}, i_{2}, \ldots, i_{n}\right\}$. The third component is a list of positive natural numbers $q_{H}=\left(q_{h_{1}}, \ldots, q_{h_{m}}\right)$, where $q_{h}$ is the total capacity of hospital $h$. Define $q_{-h}$ such that $q_{H}=\left(q_{-h}, q_{h}\right)$ for any $h \in H$. Also define $\mathcal{Q}_{h}\left(q_{h}\right)=\left\{0,1, \ldots, q_{h}\right\}$ for all $h \in H, \mathcal{Q}_{H}\left(q_{H}\right)=\times_{h \in H} \mathcal{Q}_{h}\left(q_{h}\right)$, and $\mathcal{Q}_{-h}\left(q_{-h}\right)=\times_{h^{\prime} \in H \backslash h} \mathcal{Q}_{h^{\prime}}\left(q_{h^{\prime}}\right)$ for any $h \in H$. The last component of a matching environment is a list of preference relations $R=\left(R_{h_{1}}, \ldots, R_{h_{m}}, R_{i_{1}}, \ldots, R_{i_{n}}\right)$ where $R_{h}$ is the preference relation of hospital $h$ and $R_{i}$ is the preference relation of intern $i$.

For any $h \in H, R_{h}$ is a binary preference relation that is a linear order on $\Sigma_{I}=2^{I}$. Similarly, for any $i \in I, R_{i}$ is a binary relation that is a linear order on $\Sigma_{H}=H \cup\{\emptyset\}$. Here, $\emptyset$ is interpreted by both hospitals and interns as the prospect of being unmatched.

The preference relation $R_{h}$ of hospital $h \in H$ is said to be responsive (Roth, 1985) whenever for all $I^{\prime} \subset I$ it is true that

i) for all $i \in I \backslash I^{\prime}, I^{\prime} \cup\{i\} P_{h} I^{\prime}$ if and only if $\{i\} P_{h} \emptyset$,

ii) for all $i, i^{\prime} \in I \backslash I^{\prime}, I^{\prime} \cup\{i\} P_{h} I^{\prime} \cup\left\{i^{\prime}\right\}$ if and only if $\{i\} P_{h}\left\{i^{\prime}\right\}$.

Notice that preferences of interns over the individual hospitals are trivially responsive. 
Let $\mathcal{R}_{h}$ and $\mathcal{R}_{i}$ respectively denote the class of all responsive preference relations for hospital $h \in H$ and for intern $i \in I$. Define also $\mathcal{R}=\times_{k \in H \cup I} \mathcal{R}_{k}$. Let $P_{k}$ denote the strict preference relation associated with the preference relation $R_{k}$ for agent $k \in H \cup I$. We say that for $X, Y \in\{H, I\}$ and $X \neq Y$, an element $x \in \Sigma_{X}$ is acceptable to agent $y \in Y$ if $x P_{y} \emptyset$. For each hospital $h$ define the set of acceptable interns as $A\left(R_{h}\right)=\left\{i \in I \mid\{i\} P_{h} \emptyset\right\}$, and similarly define for each intern $i$ the set of acceptable hospitals as $A\left(R_{i}\right)=$ $\left\{h \in H \mid h P_{i} \emptyset\right\}$.

Let $\mathcal{E}$ denote the set of all matching environments where the preferences are responsive.

\subsubsection{Regular Market}

Given the matching environment $E=\left(H, I, q_{H}, R\right)$, a regular market is a list $\left(E, q_{H}^{R}\right)$. The second component is a vector of nonnegative natural numbers $q_{H}^{R}=\left(q_{h_{1}}^{R}, \ldots, q_{h_{m}}^{R}\right)$, where $q_{h}^{R} \in Q_{h}\left(q_{h}\right)$ is the quota of hospital $h$ in the regular market. Define $q_{-h}^{R}$ such that $q_{H}^{R}=\left(q_{h}^{R}, q_{-h}^{R}\right)$ for any $h \in H$.

Let $\mathcal{Z}^{R}(E)$ denote the set of all possible regular markets associated with the environment $E$.

\subsubsection{Regular Market Matchings}

Given the matching environment $E$ and a regular market $Z^{R}=\left(E, q_{H}^{R}\right) \in$ $\mathcal{Z}^{R}(E)$, a matching $\mu^{R}$ in the regular market is a function from the set $H \cup I$ into $\Sigma_{I} \cup \Sigma_{H}$ such that $\mu^{R}(i) \in \Sigma_{H},\left|\mu^{R}(h)\right| \leq q_{h}^{R}$ and $\mu^{R}(i)=h$ if and only if $i \in \mu^{R}(h)$ for any $i \in I$ and $h \in H$.

We denote the set of all matchings for a given regular market $Z^{R}$ by $\mathcal{M}^{R}\left(Z^{R}\right)$. Let $\mathcal{M}^{R}=\cup_{Z^{R} \in \mathcal{Z}^{R}(E)} \mathcal{M}^{R}\left(Z^{R}\right)$.

We say that agent $k \in H \cup I$ prefers matching $\mu_{1}^{R}$ to matching $\mu_{2}^{R}$ if and only if it prefers $\mu_{1}^{R}(k)$ to $\mu_{2}^{R}(k)$ under the preference relation $R_{k}$.

Given a regular market $Z^{R}=\left(E, q_{H}^{R}\right)$, the admissible choice of hospital $h$ from a group of interns $J \subseteq I$ in the regular market is defined as

$$
\begin{gathered}
C h_{h}^{R}\left(J, Z^{R}\right)=\left\{J^{\prime} \subseteq J:\left|J^{\prime}\right| \leq q_{h}^{R}, J^{\prime} R_{h} J^{\prime \prime} \text { for all } J^{\prime \prime} \subseteq J\right. \\
\text { such that } \left.\left|J^{\prime \prime}\right| \leq q_{h}^{R}\right\} .
\end{gathered}
$$


Given a regular market $Z^{R}$, a matching $\mu^{R} \in \mathcal{M}^{R}\left(Z^{R}\right)$ is blocked by hospital $h$ if $\mu^{R}(h) \neq C h_{h}^{R}\left(\mu^{R}(h), Z^{R}\right)$. The matching $\mu^{R}$ is blocked by intern $i$ if $\emptyset P_{i} \mu^{R}(i)$. The matching $\mu^{R}$ is blocked by a hospital-intern pair $(h, i)$ in the regular market if $\{h\} P_{i} \mu^{R}(i)$, and $\mu^{R}(h) \neq C h_{h}^{R}\left(\mu^{R}(h) \cup\{i\}, Z^{R}\right)$.

A matching $\mu^{R}$ is stable if it is not blocked by an intern, a hospital, or a hospital-intern pair. We denote by $\mathcal{S}^{R}\left(Z^{R}\right)$ the set of stable matchings for the regular market $Z^{R}$.

We say, as similar in Roth and Sotomayor (1990), that hospital $h$ and intern $i$ are achievable for one another in the regular market $Z^{R}$ if there is some stable matching in $\mathcal{S}^{R}\left(Z^{R}\right)$ at which they are matched.

In the set $\mathcal{S}^{R}\left(Z^{R}\right)$ there exists a matching, $\mu_{I}^{R}$, called the intern-optimal stable matching in the regular market such that

$$
\mu_{I}^{R}(h) R_{h} \mu^{R}(h)
$$

for all $h \in H$ and for all $\mu^{R} \in \mathcal{S}^{R}\left(Z^{R}\right)$. (Analogously, one can define the hospital-optimal stable matching in the regular market.)

A regular market matching rule is a function $\varphi^{R}: \mathcal{Z}^{R}(E) \rightarrow \mathcal{M}^{R}$ such that for all $Z^{R} \in \mathcal{Z}^{R}(E)$, we have $\varphi^{R}\left(Z^{R}\right) \in \mathcal{M}^{R}\left(Z^{R}\right)$. Let $\bar{\varphi}^{R}$ denote the set of all regular market matching rules.

A regular market matching rule $\varphi^{R} \in \bar{\varphi}^{R}$ is stable if $\varphi^{R}\left(Z^{R}\right) \in \mathcal{S}^{R}\left(Z^{R}\right)$ for all $Z^{R} \in \mathcal{Z}^{R}(E)$.

\subsubsection{Aftermarket}

Given a regular market $Z^{R}=\left(E, q_{H}^{R}\right)$ and a regular market matching $\mu^{R}$ in $Z^{R}$, an aftermarket is the list $Z^{A}=\left(E, \mu^{R}\right)$. Define $\mathcal{Z}^{A}=\cup_{Z^{R} \in \mathcal{Z}^{R}(E)}$ $\cup_{\mu^{R} \in \mathcal{M}^{R}\left(Z^{R}\right)}\left(E, \mu^{R}\right)$.

\subsubsection{Aftermarket Matchings}

Given the aftermarket $Z^{A}=\left(E, \mu^{R}\right)$, we define an aftermarket matching $\mu^{A}$ as a function from the set $H \cup I$ into $\Sigma_{I} \cup \Sigma_{H}$ such that $\mu^{R}(i) \subseteq \mu^{A}(i) \in \Sigma_{H}$, $\left|\mu^{A}(h)\right| \leq q_{h}, \mu^{R}(h) \subseteq \mu^{A}(h)$ and $\mu^{A}(i)=h$ if and only if $i \in \mu^{A}(h)$ for any $i \in I$ and $h \in H$. 
We notice that the function $\mu^{A}$ preserves the matchings achieved under $\mu^{R}$ in the regular market. We denote the set of all matchings in the aftermarket $Z^{A}$ by $\mathcal{M}^{A}\left(Z^{A}\right)$. Define $\mathcal{M}^{A}=\cup_{Z^{A} \in \mathcal{Z}^{A}} \mathcal{M}^{A}\left(Z^{A}\right)$.

We say that agent $k \in H \cup I$ prefers matching $\mu_{1}^{A}$ to matching $\mu_{2}^{A}$ if and only if it prefers $\mu_{1}^{A}(k)$ to $\mu_{2}^{A}(k)$ under the preference relation $R_{k}$.

The acceptable choice of a hospital $h$ from a group of interns $J \subseteq I \backslash \mu^{R}(h)$ in the aftermarket $Z^{A}=\left(E, \mu^{R}\right)$ is defined as

$$
\begin{gathered}
C h_{h}^{A}\left(J, Z^{A}\right)=\left\{J^{\prime} \subseteq J:\left|J^{\prime}\right| \leq q_{h}-\left|\mu^{R}(h)\right|,\right. \\
J^{\prime} \cup \mu^{R}(h) R_{h} J^{\prime \prime} \cup \mu^{R}(h) \text { for all } J^{\prime \prime} \subseteq J \text { such that } \\
\left.\left|J^{\prime \prime}\right| \leq q_{h}-\left|\mu^{R}(h)\right|\right\} .
\end{gathered}
$$

Given the aftermarket $Z^{A}=\left(E, \mu^{R}\right)$, a matching $\mu^{A} \in \mathcal{M}^{A}\left(Z^{A}\right)$ is blocked by hospital $h$ if $\mu^{A}(h) \backslash \mu^{R}(h) \neq C h_{h}^{A}\left(\mu^{A}(h) \backslash \mu^{R}(h), Z^{A}\right)$. The matching $\mu^{A}$ is blocked by an intern $i$ if $\emptyset P_{i} \mu^{A}(i) \backslash \mu^{R}(i)$. The matching $\mu^{A}$ is blocked by a hospital-intern pair $(h, i)$ in the aftermarket if $\mu^{R}(i)=\emptyset$, $\{h\} P_{i} \mu^{A}(i)$ and $\mu^{A}(h) \backslash \mu^{R}(h) \neq C h_{h}\left(\{i\} \cup \mu^{A}(h) \backslash \mu^{R}(h), Z^{A}\right)$.

A matching $\mu^{A}$ is stable at $Z^{A}$ if it is not blocked by an intern, a hospital, or a hospital-intern pair. We denote by $\mathcal{S}^{A}\left(Z^{A}\right)$ the set of matchings that are stable at $Z^{A}$.

We say that hospital $h$ and intern $i$ are achievable for one another in the aftermarket $Z^{A}$ if there is some stable matching in $\mathcal{S}^{A}\left(Z^{A}\right)$ at which they are matched.

In the set $\mathcal{S}^{A}\left(Z^{A}\right)$, there exists a matching, $\mu_{I}^{A}$, called the intern-optimal stable matching in the aftermarket such that

$$
\mu_{I}^{A}(h) R_{h} \mu^{A}(h)
$$

for all $h \in H$ and for all $\mu^{A} \in \mathcal{S}^{A}\left(Z^{A}\right){ }^{4}$ (Analogously, one can define the hospital-optimal stable matching in the aftermarket.)

\footnotetext{
${ }^{4}$ To find the intern-optimal stable matching in the regular market and aftermarket, we respectively use the well-known intern-proposing deferred acceptance algorithm by Gale and Shapley (1962).
} 
An aftermarket matching rule is a function $\varphi^{A}: \mathcal{Z}^{A} \rightarrow \mathcal{M}^{A}$ such that for all $Z^{A} \in \mathcal{Z}^{A}$, we have $\varphi^{A}\left(Z^{A}\right) \in \mathcal{M}^{A}\left(Z^{A}\right)$. Let $\bar{\varphi}^{A}$ denote the set of all aftermarket matching rules.

An aftermarket matching rule $\varphi^{A} \in \bar{\varphi}^{A}$ is stable at $Z^{A}$ if $\varphi^{A}\left(Z^{A}\right) \in$ $\mathcal{S}^{A}\left(Z^{A}\right)$.

\subsubsection{Matching Systems}

For any $\varphi^{R} \in \bar{\varphi}^{R}$ that is used in the regular market and for any $\varphi^{A} \in \bar{\varphi}^{A}$ that is used in the aftermarket, the ordered pair $\left(\varphi^{R}, \varphi^{A}\right)$ is called a matching system. Let $\vec{\varphi}$ denote the matching system $\left(\varphi^{R}, \varphi^{A}\right)$.

A matching system $\vec{\varphi}$ is stable if (i) $\varphi^{R}$ is stable, and (ii) for any $Z^{R} \in$ $\mathcal{Z}^{R}(E)$ and $\mu^{R} \in \mathcal{M}^{R}\left(Z^{R}\right), \varphi^{A}$ is stable at $\left(E, \mu^{R}\right)$.

Let $\vec{\varphi}_{I}$ be a matching system such that $\varphi_{I}^{R}\left(Z^{R}\right)=\mu_{I}^{R}$ for all $Z^{R} \in \mathcal{Z}^{R}(E)$ and $\varphi_{I}^{A}\left(Z^{A}\right)=\mu_{I}^{A}$ for all $Z^{A} \in \mathcal{Z}^{A}$. We call $\vec{\varphi}_{I}$ as the intern-optimal stable matching system. (Analogously, one can define the hospital-optimal stable matching system.)

\subsection{A Game of Capacity Allocation}

We consider a game of capacity allocation played by hospitals in a given hospital-intern matching environment $E=\left(H, I, q_{H}, R\right)$.

Each hospital is asked to announce out of its total capacity a regular market quota; formally, the strategy of hospital $h$ is $q_{h}^{R} \in \mathcal{Q}_{h}\left(q_{h}\right)$. We assume that for each possible announcement of the vector $q_{H}^{R}$, the regular market and the aftermarket are common knowledge. Suppose that a matching system $\vec{\varphi}$ is used to determine the matchings in the regular market and the aftermarket. Hospital $h$ 's preferences over the reported regular market quotas are represented by a binary relationship $\succeq_{h}^{\vec{\varphi}}$ over $\mathcal{Q}_{H}\left(q_{H}\right)$ such that for all $q^{\prime}, q^{\prime \prime} \in \mathcal{Q}_{H}\left(q_{H}\right)$ we have $q^{\prime} \succeq_{h}^{\vec{\varphi}} q^{\prime \prime}$ if and only if

$$
\varphi^{A}\left(E, \varphi^{R}\left(E, q^{\prime}\right)\right)(h) R_{h} \varphi^{A}\left(E, \varphi^{R}\left(E, q^{\prime \prime}\right)\right)(h) .
$$

A game of capacity allocation under matching system $\vec{\varphi}$ is described by a strategic form game $\left\langle H,\left(\mathcal{Q}_{h}\left(q_{h}\right), \succeq_{h}^{\vec{\varphi}}\right)_{h \in H}\right\rangle$. 
Define hospital $h$ 's best response correspondence under matching system $\vec{\varphi}$ by $\beta_{h}^{\vec{\varphi}}: \mathcal{Q}_{-h}\left(q_{-h}\right) \rightarrow \mathcal{Q}_{h}\left(q_{h}\right)$ such that for any $q_{-h}^{\prime} \in \mathcal{Q}_{-h}\left(q_{-h}\right)$,

$$
\beta_{h}^{\vec{\varphi}}\left(q_{-h}^{\prime}\right)=\left\{q_{h}^{\prime} \in \mathcal{Q}_{h}\left(q_{h}\right):\left(q_{h}^{\prime}, q_{-h}^{\prime}\right) \succeq_{h}^{\varphi}\left(\tilde{q}_{h}, q_{-h}^{\prime}\right) \text { for all } \tilde{q}_{h} \in \mathcal{Q}_{h}\left(q_{h}\right)\right\} .
$$

A pure-strategy (Nash) equilibrium of the game $\left\langle H,\left(\mathcal{Q}_{h}\left(q_{h}\right), \succeq_{h}^{\vec{\varphi}}\right)_{h \in H}\right\rangle$ is a strategy profile $q_{H}^{*} \in \mathcal{Q}_{H}\left(q_{H}\right)$ such that $q_{h}^{*} \in \beta_{h}^{\vec{\varphi}}\left(q_{-h}^{*}\right)$ for all $h \in H$.

\section{Results}

The following theorem shows that a pure-strategy equilibrium may not exist under the intern-optimal stable matching system. ${ }^{5}$

Theorem 1. The capacity allocation game under the intern-optimal stable matching system may not have a pure-strategy equilibrium.

Proof. Consider the environment $\left(H, I, q_{H}, R\right)$ with $H=\left\{h_{1}, h_{2}\right\}, I=$ $\left\{i_{1}, i_{2}, i_{3}, i_{4}, i_{5}\right\}, q_{h_{1}}=3, q_{h_{2}}=3$,

$$
\begin{aligned}
& P_{h_{1}}=\left\{i_{1}\right\},\left\{i_{2}\right\},\left\{i_{5}\right\},\left\{i_{3}\right\},\left\{i_{4}\right\}, \emptyset, \text { with }\left\{i_{2}, i_{3}\right\} P_{h_{1}}\left\{i_{1}, i_{4}\right\}, \\
& P_{h_{2}}=\left\{i_{2}\right\},\left\{i_{5}\right\},\left\{i_{1}\right\},\left\{i_{4}\right\},\left\{i_{3}\right\}, \emptyset, \text { with }\left\{i_{2}, i_{3}\right\} P_{h_{2}}\left\{i_{1}, i_{4}\right\},
\end{aligned}
$$

and $I^{\prime} P_{h} I^{\prime \prime}$ for any $h \in H$ and $I^{\prime}, I^{\prime \prime} \subseteq I$ such that $\left|I^{\prime}\right|>\left|I^{\prime \prime}\right|$,

$$
\begin{aligned}
& P_{i_{1}}=\left\{h_{2}\right\},\left\{h_{1}\right\}, \emptyset, \\
& P_{i_{2}}=\left\{h_{1}\right\},\left\{h_{2}\right\}, \emptyset, \\
& P_{i_{3}}=\left\{h_{2}\right\},\left\{h_{1}\right\}, \emptyset, \\
& P_{i_{4}}=\left\{h_{1}\right\},\left\{h_{2}\right\}, \emptyset, \\
& P_{i_{5}}=\left\{h_{2}\right\},\left\{h_{1}\right\}, \emptyset .
\end{aligned}
$$

We have $\mathcal{Q}_{h}\left(q_{h}\right)=\{0,1,2,3\}$ for any $h \in H$.

\footnotetext{
${ }^{5}$ We restrict our attention to the intern-optimal stable matching rule (matching system in our two-stage model) since it is currently used by the NRMP for regular hospital-intern matchings in the USA.
} 
Under the intern-optimal stable matching system $\vec{\varphi}_{I}$, Table 1 below gives the outcome allocations in the regular market for each pure-strategy profile $q_{H}^{R}$ in $\mathcal{Q}_{H}\left(q_{H}\right)$. The first entry in each cell is the assignment of $h_{1}$ and the second entry is the assignment of $h_{2}$. Those interns who are not assigned to either $h_{1}$ or $h_{2}$ remain unemployed in the regular market.

Table 1.

\begin{tabular}{|c|c|c|c|c|}
\hline & $q_{h_{2}}^{R}=0$ & $q_{h_{2}}^{R}=1$ & $q_{h_{2}}^{R}=2$ & $q_{h_{2}}^{R}=3$ \\
\hline$q_{h_{1}}^{R}=0$ & $\emptyset, \emptyset$ & $\emptyset,\left\{i_{2}\right\}$ & $\emptyset,\left\{i_{2}, i_{5}\right\}$ & $\emptyset,\left\{i_{1}, i_{2}, i_{5}\right\}$ \\
\hline$q_{h_{1}}^{R}=1$ & $\left\{i_{1}\right\}, \emptyset$ & $\left\{i_{1}\right\},\left\{i_{2}\right\}$ & $\left\{i_{2}\right\},\left\{i_{1}, i_{5}\right\}$ & $\left\{i_{2}\right\},\left\{i_{1}, i_{4}, i_{5}\right\}$ \\
\hline$q_{h_{1}}^{R}=2$ & $\left\{i_{1}, i_{2}\right\}, \emptyset$ & $\left\{i_{1}, i_{2}\right\},\left\{i_{5}\right\}$ & $\left\{i_{2}, i_{3}\right\},\left\{i_{1}, i_{5}\right\}$ & $\left\{i_{2}, i_{4}\right\},\left\{i_{1}, i_{3}, i_{5}\right\}$ \\
\hline$q_{h_{1}}^{R}=3$ & $\left\{i_{1}, i_{2}, i_{5}\right\}, \emptyset$ & $\left\{i_{1}, i_{2}, i_{3}\right\},\left\{i_{5}\right\}$ & $\left\{i_{2}, i_{3}, i_{4}\right\},\left\{i_{1}, i_{5}\right\}$ & $\left\{i_{2}, i_{4}\right\},\left\{i_{1}, i_{3}, i_{5}\right\}$ \\
\hline
\end{tabular}

In Table 2, we give the outcome allocations in the aftermarket for each pure-strategy profile $q_{H}^{R} \in \mathcal{Q}_{H}\left(q_{H}\right)$ given the aftermarket matching rule $\varphi_{I}^{A}$.

Table 2.

\begin{tabular}{|c|c|c|c|c|}
\hline & $q_{h_{2}}^{R}=0$ & $q_{h_{2}}^{R}=1$ & $q_{h_{2}}^{R}=2$ & $q_{h_{2}}^{R}=3$ \\
\hline$q_{h_{1}}^{R}=0$ & $\left\{i_{2}, i_{4}\right\},\left\{i_{1}, i_{3}, i_{5}\right\}$ & $\left\{i_{3}, i_{4}\right\},\left\{i_{1}, i_{2}, i_{5}\right\}$ & $\left\{i_{3}, i_{4}\right\},\left\{i_{1}, i_{2}, i_{5}\right\}$ & $\left\{i_{3}, i_{4}\right\},\left\{i_{1}, i_{2}, i_{5}\right\}$ \\
\hline$q_{h_{1}}^{R}=1$ & $\left\{i_{1}, i_{2}, i_{4}\right\},\left\{i_{3}, i_{5}\right\}$ & $\left\{i_{1}, i_{4}\right\},\left\{i_{2}, i_{3}, i_{5}\right\}$ & $\left\{i_{2}, i_{4}\right\},\left\{i_{1}, i_{3}, i_{5}\right\}$ & $\left\{i_{2}, i_{3}\right\},\left\{i_{1}, i_{4}, i_{5}\right\}$ \\
\hline$q_{h_{1}}^{R}=2$ & $\left\{i_{1}, i_{2}, i_{4}\right\},\left\{i_{3}, i_{5}\right\}$ & $\left\{i_{1}, i_{2}, i_{4}\right\},\left\{i_{3}, i_{5}\right\}$ & $\left\{i_{2}, i_{3}, i_{4}\right\},\left\{i_{1}, i_{5}\right\}$ & $\left\{i_{2}, i_{4}\right\},\left\{i_{1}, i_{3}, i_{5}\right\}$ \\
\hline$q_{h_{1}}^{R}=3$ & $\left\{i_{1}, i_{2}, i_{5}\right\},\left\{i_{3}, i_{4}\right\}$ & $\left\{i_{1}, i_{2}, i_{3}\right\},\left\{i_{4}, i_{5}\right\}$ & $\left\{i_{2}, i_{3}, i_{4}\right\},\left\{i_{1}, i_{5}\right\}$ & $\left\{i_{2}, i_{4}\right\},\left\{i_{1}, i_{3}, i_{5}\right\}$ \\
\hline
\end{tabular}

According to the preferences of hospitals, their best response correspondences are:

$$
\begin{gathered}
\beta_{h_{1}}^{\vec{\varphi}_{H}}(0)=\{3\}, \beta_{h_{1}}^{\vec{\varphi}_{H}}(1)=\{3\}, \beta_{h_{1}}^{\vec{\varphi}_{H}}(2)=\{2,3\}, \beta_{h_{1}}^{\vec{\varphi}_{H}}(3)=\{1\}, \\
\beta_{h_{2}}^{\vec{\varphi}_{H}}(0)=\{1,2,3\}, \beta_{h_{2}}^{\vec{\varphi}_{H}}(1)=\{1\}, \beta_{h_{2}}^{\vec{\varphi}_{H}}(2)=\{3\}, \beta_{h_{2}}^{\vec{\varphi}_{H}}(3)=\{3\} .
\end{gathered}
$$

Therefore the game has no pure-strategy equilibrium.

Konishi and Ünver (2006) have a similar result showing the failure of the general existence of pure-strategy equilibria of games of capacity manipulation in single-period hospital-intern markets. They also argue that hospitals can improve their position by restricting the available capacity under the 
hospital-optimal matching rule. The reason is that larger capacities of hospitals make interns better off by giving them more alternatives to choose from. However, when capacities are limited, an intern cannot play one offer against the other and has to choose among rather limited set of offers which may not include his/her favorite hospital. Konishi and Ünver (2006) additionally remark that hospitals, which are getting their least preferable acceptable interns under the intern-optimal matching rule, can do better by swapping interns through some reduction in their quotas. In our model, similar incentives are at work. However, given their fixed total capacities hospitals are now able to improve their position not by limiting the total number of incoming interns but by spreading the offering process across periods. This essentially increases their "bargaining power" without necessarily reducing the overall intake of the interns. If we use the analogy between the hospitalintern market and the marriage market, as remarked by Roth (1985), by viewing each vacant position in a hospital as an individual player who has the same preferences as the hospital that it belongs to, spreading admission across periods increases the bargaining power of a hospital by making the supply side of the market "thinner".

Another interesting finding (capacity lemma) of Konishi and Ünver (2006) is that a hospital's capacity underreport makes all other hospitals weakly better off and all interns weakly worse off under both hospital-optimal stable matching and intern-optimal stable matching. But, this observation does not carry over our framework that involves two stages of admission. ${ }^{6}$

Remark 1. In games of capacity allocation, a hospital's regular market quota report in pure strategies does not have a monotonic effect on the welfare of other hospitals and interns under the intern-optimal stable matching system.

Proof. Consider the example in the proof of Theorem 1. From Table 2, if $h_{1}$ decreases its regular market quota at $q_{H}^{R}=(3,1), h_{2}$ is first worse off since

\footnotetext{
${ }^{6}$ Our propositions in Theorem 1 and Remark 1 readily extend to matching environments that employ the hospital-optimal stable matching system.
} 
$\left\{i_{4}, i_{5}\right\} P_{h_{2}}\left\{i_{3}, i_{5}\right\}$, and then better off since $\left\{i_{2}, i_{3}, i_{5}\right\} P_{h_{2}}\left\{i_{3}, i_{5}\right\}$. In terms of the interns' welfare, we observe that in Table 2 , if $h_{1}$ reduces its regular market quota at $q_{H}^{R}=(3,1)$, not all interns are weakly worse off. For example, at $q_{H}^{R}=(0,1), i_{1}$ is better off whereas $i_{2}$ is worse off.

Given the nonexistence of equilibrium in pure strategies, a natural reaction is to allow mixed strategies in games of capacity allocation. Along a similar reflection on the nonexistence result in Konishi and Ünver (2006), Kojima (2006) shows that a mixed-strategy equilibrium always exists in games of capacity manipulation in hospital-intern markets. Kojima (2006) further shows that the aforementioned capacity lemma of Konishi and Ünver (2006) can be generalized in the presence of mixed strategies.

Here, we define a mixed strategy of a hospital with total capacity $q_{h}$ as a probability distribution on the set of possible regular market quotas. Closely following the definitions in Kojima (2006), a mixed strategy of $h$ is $p_{h}=\left(p_{h}^{0}, p_{h}^{1}, \ldots, p_{h}^{q_{h}}\right) \in \Delta_{h} \equiv\left\{\left(p_{h}^{0}, p_{h}^{1}, \ldots, p_{h}^{q_{h}}\right) \in \mathbb{R}^{q_{h}} ; p_{h}^{j} \geq 0, \sum_{j} p_{h}^{j}=1\right\}$, where $p_{h}^{j}$ is the probability that $h$ reports that its regular market quota is $j$. We assume that the preferences of each hospital and intern satisfy the vonNeumann Morgenstern expected utility hypothesis. Given a matching environment $E=\left(H, I, q_{H}, R\right)$ and the intern-optimal stable matching system $\vec{\varphi}_{I}$, we denote by $\varphi_{I}^{A}\left(E, \varphi_{I}^{R}\left(E, p_{H}\right)\right)$ a distribution on aftermarket matchings induced by the mixed-strategy profile $p_{H}$. We also assume that preferences over matchings directly extend to preferences over distributions on matchings for both hospitals and interns. Finally, we say that of the two distinct quota profiles $p_{H}$ and $\tilde{p}_{H}$, the latter is a larger quota profile if

$$
\sum_{s \geq j} p_{h}^{s} \leq \sum_{s \geq j} \tilde{p}_{h}^{s} \text { for any } h \in H \text { and } j \in Q_{h}\left(q_{h}\right) .
$$

It is immediate from Nash (1951) that there always exists a Nash equilibrium in mixed strategies in games of capacity allocation. However, we do not expect like in the generalization result of Kojima (2006) that every hospital will always weakly prefer a non-degenerate mixed- strategy Nash equilibrium to any larger capacity profiles, owing to an insight from Remark 1 that over the supports of some mixed-strategy equilibria a hospital's underreporting 
its regular market quota may not increase the welfare of the other hospitals in the market.

Remark 2. For games of capacity allocation, it is not true that every hospital weakly prefers a Nash equilibrium (pure or mixed) to any larger regular market quota profiles like in games of capacity manipulation.

Proof. Given that Remark 1 deals with pure strategies, we only need to consider non-degenerate mixed strategies. We use the example in the proof of Theorem 1, which was verified to have no Nash equilibrium in pure strategies. Below table assigns utility payoffs for each outcome in Table 2 in such a way that the ordinal preferences of the hospitals are preserved.

Table 3.

\begin{tabular}{|c|c|c|c|c|}
\hline & $q_{h_{2}}^{R}=0$ & $q_{h_{2}}^{R}=1$ & $q_{h_{2}}^{R}=2$ & $q_{h_{2}}^{R}=3$ \\
\hline$q_{h_{1}}^{R}=0$ & $(8,20)$ & $(4,32)$ & $(4,32)$ & $(4,32)$ \\
\hline$q_{h_{1}}^{R}=1$ & $(26,8)$ & $(12,28)$ & $(8,20)$ & $(24,24)$ \\
\hline$q_{h_{1}}^{R}=2$ & $(26,8)$ & $(26,8)$ & $(25,16)$ & $(8,20)$ \\
\hline$q_{h_{1}}^{R}=3$ & $(32,4)$ & $(28,12)$ & $(25,16)$ & $(8,20)$ \\
\hline
\end{tabular}

One can check that the profile $p_{H}$, where $p_{h_{1}}=(0,2 / 3,0,1 / 3)$ and $p_{h_{2}}=$ $(0,1 / 2,0,1 / 2)$, is a mixed-strategy equilibrium. The expected payoffs of $h_{1}$ and $h_{2}$ at the quota profile $p_{H}$ are calculated as 18 and 68/3, respectively. Now consider a larger quota profile $\tilde{p}_{H}$ such that $\tilde{p}_{h_{1}}=p_{h_{1}}$ and $\tilde{p}_{h_{2}}=(0,0,0,1)$. The expected payoffs of $h_{1}$ and $h_{2}$ at the quota profile $\tilde{p}_{H}$ are calculated as 56/3 and 68/3, respectively. Hence, the claim in Remark 2 follows.

To cure the failure of general existence of equilibrium in pure strategies, we will now consider preference restrictions. Dealing with a similar problem in single-stage hospital-intern markets, Konishi and Ünver (2006) consider two types of restrictions. In one of them, hospitals' preferences are said to satisfy strong monotonicity in population if any hospital strictly prefers among any two groups of acceptable interns of distinct sizes the one that 
is more populated. With this domain restriction, Konishi and Ünver (2006) show that in the capacity-reporting game, under the hospital-optimal matching rule reporting the number of interns that the matching rule assigns is an equilibrium strategy whereas under the intern-optimal matching rule reporting the actual capacity is a weakly dominant strategy.

In our model, we say that hospitals' preferences $R \in \mathcal{R}$ satisfy strong monotonicity in population, if for any $h \in H$ and for any $I^{\prime}, I^{\prime \prime} \subseteq I$ such that any $i \in I^{\prime} \cup I^{\prime \prime}$ is acceptable to $h$, we have $\left|I^{\prime}\right|>\left|I^{\prime \prime}\right|$ only if $I^{\prime} P_{h} I^{\prime \prime}$.

We should immediately note that the preferences of hospitals in the matching environment considered in the proof of Theorem 1 already satisfy strong monotonicity in population. ${ }^{7}$ Therefore, we conclude that:

Remark 3. Strong monotonicity in population is not a sufficient restriction on hospitals' preferences to ensure the existence of equilibrium in pure strategies in games of capacity allocation.

The second type of restriction that Konishi and Ünver (2006) consider is the common preferences for one group over the agents of the opposite group. With such preferences, they are able to show that reporting the true capacity is always a weakly dominant strategy. Below, we will show that this kind of domain restriction will also allow us to achieve an existence result.

A preference profile $R \in \mathcal{R}$ satisfies common preferences for hospitals over individual interns if and only if for any $h, h^{\prime} \in H$ and for any $i, i^{\prime} \in I$ we have $\{i\} P_{h}\left\{i^{\prime}\right\} \Leftrightarrow\{i\} P_{h^{\prime}}\left\{i^{\prime}\right\} .8$

Let $\mathcal{R}^{C} \subset \mathcal{R}$ be the domain of such profiles of common preference relations.

Now, pick $R \in \mathcal{R}^{C}$, and consider the environment $E=\left(H, I, q_{H}, R\right)$. Rename and reorder acceptable interns by the preference ordering $P_{h}$ of hospital

\footnotetext{
${ }^{7}$ Remark 3 is also obtained for matching environments that employ the hospital-optimal stable matching system.

${ }^{8}$ This definition of common preferences for hospitals weakens a respective definition in Konishi and Ünver (2006) that also requires a common set of acceptable interns under the common preferences for hospitals.
} 
$h$ as $i_{1}, i_{2}, \ldots, i_{l(h)}$. That is, hospital $c$ has the preference ordering

$$
P_{h}=\left\{i_{1}\right\},\left\{i_{2}\right\}, \ldots,\left\{i_{l(h)}\right\}, \emptyset .
$$

Let $\hat{l}=\max \{l(h): h \in H\}$. Define $C h_{i}\left(R_{i}, H^{\prime}\right)$ be the most preferable acceptable hospital in $H^{\prime} \subseteq H$ for intern $i$ with respect to the preference relation $R_{i}$, i.e., $C h_{i}\left(R_{i}, H^{\prime}\right)=\left\{h \in H^{\prime} \cap A\left(R_{i}\right):\{h\} R_{i}\left\{h^{\prime}\right\}\right.$ for any $\left.h^{\prime} \in H^{\prime}\right\}$. If $\hat{l}>0$, consider a matching $\hat{\mu}^{R}$ in the regular market generated by the following serial-dictatorship: ${ }^{9}$

Step 1: Let $H^{R, 1}=\left\{h \in H: q_{h}^{R} \neq 0\right.$ and $\left.i_{1} \in A\left(R_{h}\right)\right\}$ and $q_{h}^{R, 1}=q_{h}^{R}$ for all $h \in H$. Set $\hat{\mu}^{R}\left(i_{1}\right)=C h_{i_{1}}^{R}\left(R_{i_{1}}, H^{R, 1}\right)$.

Step t: For all $h \in H$, let $q_{h}^{R, t}=q_{h}^{R, t-1}-1$ if $\hat{\mu}^{R}\left(i_{t-1}\right)=\{h\}$, and $q_{h}^{R, t}=q_{h}^{R, t-1}$ otherwise. Let $H^{R, t}=\left\{h \in H: q_{h}^{R, t} \neq 0\right.$ and $\left.i_{t} \in A\left(R_{h}\right)\right\}$. Set $\hat{\mu}^{R}\left(i_{t}\right)=$ $C h_{i_{t}}^{R}\left(R_{i_{t}}, H^{R, t}\right)$.

The above algorithm stops after $\hat{l}$ steps, and $\hat{\mu}^{R}$ becomes the matching of the regular market. Notice that for all $i \in I \backslash\left\{i_{1}, i_{2}, \ldots, i_{\hat{l}}\right\}$, we have $\hat{\mu}^{R}(i)=\emptyset$, trivially. If $\hat{l}=0$, set $\hat{\mu}^{R}(i)=\emptyset$ for all $i \in I$.

Now, we consider a similar matching in the aftermarket. Given the matching $\hat{\mu}^{R}$, consider a matching $\hat{\mu}^{A}$ in the aftermarket, generated by the following serial-dictatorship if $\hat{l}>0$ :

Step 1: Let $q_{h}^{A, 1}\left(\hat{\mu}^{R}\right)=q_{h}-\left|\hat{\mu}^{R}(h)\right|$ for all $h \in H$, and $H^{A, 1}\left(\hat{\mu}^{R}\right)=\{h \in$ $H: q_{h}^{A, 1}\left(\hat{\mu}^{R}\right) \neq 0$ and $\left.i_{1} \in A\left(R_{h}\right)\right\}$. Set $\hat{\mu}^{A}\left(i_{1}\right)=C h_{i_{1}}\left(R_{i_{1}}, H^{A, 1}\left(\hat{\mu}^{R}\right)\right)$ if $\hat{\mu}^{R}\left(i_{1}\right)=\emptyset$, and $\hat{\mu}^{A}\left(i_{1}\right)=\hat{\mu}^{R}\left(i_{1}\right)$ otherwise.

Step t: For all $h \in H$, let $q_{h}^{A, t}\left(\hat{\mu}^{R}\right)=q_{h}^{A, t-1}\left(\hat{\mu}^{R}\right)-1$ if $\hat{\mu}^{A}\left(i_{t-1}\right) \backslash \hat{\mu}^{R}\left(i_{t-1}\right)=\{h\}$,

\footnotetext{
${ }^{9}$ The serial dictatorship rules that we use extend respective rules in Konishi and Ünver (2006) to our two-stage framework. For the other uses of serial dictatorship in one-sided matching markets, see Svensson (1994), Abdulkadirog̃lu and Sönmez (1998), and Papai (2000).
} 
and $q_{h}^{A, t}\left(\hat{\mu}^{R}\right)=q_{h}^{A, t-1}\left(\hat{\mu}^{R}\right)$ otherwise. Let $H^{A, t}\left(\hat{\mu}^{R}\right)=\left\{h \in H: q_{h}^{A, t}\left(\hat{\mu}^{R}\right) \neq\right.$ 0 and $\left.i_{t} \in A\left(R_{h}\right)\right\}$. Set $\hat{\mu}^{A}\left(i_{t}\right)=C h_{i_{t}}\left(R_{i_{t}}, H^{A, t}\left(\hat{\mu}^{R}\right)\right)$ if $\hat{\mu}^{R}\left(i_{t}\right)=\emptyset$, and $\hat{\mu}^{A}\left(i_{t}\right)=\hat{\mu}^{R}\left(i_{t}\right)$ otherwise.

The above algorithm stops after $\hat{l}$ steps, and $\hat{\mu}^{A}$ becomes the matching of the aftermarket. Notice that for all $i \in I \backslash\left\{i_{1}, i_{2}, \ldots, i_{\hat{l}}\right\}$, we have $\hat{\mu}^{A}(i)=\hat{\mu}^{R}(i)=\emptyset$, trivially. If $\hat{l}=0$, set $\hat{\mu}^{A}(i)=\hat{\mu}^{R}(i)=\emptyset$ for all $i \in I$.

Lemma 1. Consider any hospital-intern market with common preferences for hospitals. Then $\left(\hat{\mu}^{R}, \hat{\mu}^{A}\right)$ is the unique pair of stable matchings applied in the regular market and aftermarket, respectively.

Proof. Here, we extend the proof of Lemma 2 in Konishi and Ünver (2006). Consider any matching environment $E=\left(H, I, q_{H}, R\right)$, where $R \in \mathcal{R}^{C}$. Let $q_{H}^{R} \in \mathcal{Q}_{H}\left(q_{H}\right)$. We will show that $\left(\hat{\mu}^{E}, \hat{\mu}^{R}\right)$ is the unique pair of stable matchings in the early and regular decision periods.

Consider any stable matching $\mu^{R}$ in the regular market. If $\hat{l}=0$, then $\mu^{R}(i)=\emptyset$ for all $i \in I$. If $\hat{l}>0$, we have $\mu^{R}\left(i_{1}\right)=C h_{i_{1}}^{R}\left(R_{i_{1}}, H^{R, 1}\right)$, for otherwise the pair $\left(i_{1}, C h_{i_{1}}^{R}\left(R_{i_{1}}, H^{R, 1}\right)\right)$ would block $\mu^{R}$ if $C h_{i_{1}}^{R}\left(R_{i_{1}}, H^{R, 1}\right) \neq \emptyset$; and $i_{1}$ would be better off by staying unmatched if $C h_{i_{1}}^{R}\left(R_{i_{1}}, H^{R, 1}\right)=\emptyset$. If $\hat{l}>1$, first note that $H^{R, t} \supseteq H^{R, t+1}$ for any $t \leq \hat{l}-1$, since $H^{R, t}$ monotonically shrinks (weakly) by construction. Then, given $\mu^{R}\left(i_{1}\right)$, we have $\mu^{R}\left(i_{2}\right)=$ $C h_{i_{2}}^{R}\left(R_{i_{2}}, H^{R, 2}\right)$, for otherwise the pair $\left(i_{2}, C h_{i_{2}}^{R}\left(R_{i_{2}}, H^{R, 2}\right)\right)$ would block $\mu^{R}$ if $C h_{i_{2}}^{R}\left(R_{i_{2}}, H^{R, 2}\right) \neq \emptyset$; and $i_{2}$ would be better off by staying unmatched if $C h_{i_{2}}^{R}\left(R_{i_{2}}, H^{R, 2}\right)=\emptyset$. Similarly, for any $t \leq \hat{l}, \mu^{R}\left(i_{t}\right)=C h_{i_{t}}^{R}\left(R_{i_{t}}, H^{R, t}\right)$. Thus, we must have $\mu^{R}=\hat{\mu}^{R}$.

Now given $\hat{\mu}^{R}$, consider any stable matching $\mu^{A}$ in the aftermarket. If $\hat{l}=0$, then $\mu^{A}(i)=\hat{\mu}^{R}(i)=\emptyset$ for all $i \in I$. Now consider the case in which $\hat{l}>0$. Clearly, $\mu^{A}(i)=\hat{\mu}^{R}(i)$ for all $i \in\left\{i_{1}, i_{2}, \ldots, i_{\hat{l}}\right\}$ such that $\hat{\mu}^{R}(i) \neq \emptyset$. Moreover, we have $\mu^{A}\left(i_{1}\right)=C h_{i_{1}}^{A}\left(R_{i_{1}}, H^{A, 1}\left(\hat{\mu}^{R}\right)\right)$ if $\hat{\mu}^{R}\left(i_{1}\right)=\emptyset$, for otherwise the pair $\left(i_{1}, C h_{i_{1}}^{A}\left(R_{i_{1}}, H^{A, 1}\left(\hat{\mu}^{R}\right)\right)\right)$ would block $\mu^{A}$ if $C h_{i_{1}}^{A}\left(R_{i_{1}}, H^{A, 1}\left(\hat{\mu}^{R}\right)\right) \neq \emptyset$; and $i_{1}$ would be better off by staying unmatched if $C h_{i_{1}}^{A}\left(R_{i_{1}}, H^{A, 1}\left(\hat{\mu}^{R}\right)\right)=$ $\emptyset$. If $\hat{l}>1$, first note that $H^{A, t}\left(\hat{\mu}^{R}\right) \supseteq H^{A, t+1}\left(\hat{\mu}^{R}\right)$ for any $t \leq \hat{l}-1$, since $H^{A, t}\left(\hat{\mu}^{R}\right)$ monotonically shrinks (weakly) by construction. Then, given 
$\mu^{A}\left(i_{1}\right)$, we have $\mu^{A}\left(i_{2}\right)=C h_{i_{2}}^{A}\left(R_{i_{2}}, H^{A, 2}\left(\hat{\mu}^{R}\right)\right)$ if $\hat{\mu}^{R}\left(i_{2}\right)=\emptyset$, for otherwise the pair $\left(i_{2}, C h_{i_{2}}^{A}\left(R_{i_{2}}, H^{A, 2}\left(\hat{\mu}^{R}\right)\right)\right)$ would block $\mu^{A}$ if $C h_{i_{2}}^{A}\left(R_{i_{2}}, H^{A, 2}\left(\hat{\mu}^{R}\right)\right) \neq \emptyset$; and $i_{2}$ would be better off by staying unmatched if $C h_{i_{2}}^{A}\left(R_{i_{2}}, H^{A, 2}\left(\hat{\mu}^{R}\right)\right)=\emptyset$. Similarly, for any $t \leq \hat{l}, \mu^{A}\left(i_{t}\right)=C h_{i_{t}}^{A}\left(R_{i_{t}}, H^{A, t}\left(\hat{\mu}^{R}\right)\right)$ if $\hat{\mu}^{R}\left(i_{t}\right)=\emptyset$. Thus, we must have $\mu^{A}=\hat{\mu}^{A}$.

Below, we show that reporting the total capacity in the regular market is a weakly dominant strategy for each hospital. ${ }^{10}$

Theorem 2. Consider a matching environment with common preferences for hospitals. Then, in the capacity allocation game under the stable matching system, reporting the total capacity as the regular market quota is a weakly dominant strategy for each hospital.

Proof. Consider any matching environment $E=\left(H, I, q_{H}, R\right)$, where $R \in \mathcal{R}^{C}$. Let $q_{H}^{R} \in \mathcal{Q}_{H}\left(q_{H}\right)$. Pick a hospital $h \in H$, and consider a strategy $q_{H}^{\prime} \in \mathcal{Q}_{H}\left(q_{H}\right)$. Let $\overrightarrow{\hat{\varphi}}$ be the unique stable matching system associated with the regular market $\left(E,\left(q_{h}, q_{-h}^{\prime}\right)\right)$. Let $\varphi^{A}\left(E, \varphi^{R}\left(E,\left(q_{h}, q_{-h}^{\prime}\right)\right)\right)(h)=$ $\varphi^{R}\left(E,\left(q_{h}, q_{-h}^{\prime}\right)\right)=\left\{i_{k}, i_{l}, \ldots, i_{r}\right\}$, where $k<l<\ldots<r \leq n$. For any $p<r$, $i_{p} \notin \hat{\varphi}^{A}\left(E, \varphi^{R}\left(E, q_{H}^{\prime}\right)\right)(h)$ if $i_{p} \notin\left\{i_{k}, i_{l}, \ldots, i_{r}\right\}$, following by the construction of $\hat{\varphi}^{R}$ and $\hat{\varphi}^{A}$. Moreover, $\left.\varphi^{R}\left(E, q_{H}^{\prime}\right)\right)=T^{R} \subseteq\left\{i_{k}, i_{l}, \ldots, i_{r}\right\}$ where $\left|T^{R}\right| \leq q_{h}^{\prime}$, and $\hat{\varphi}^{A}\left(E, \varphi^{R}\left(E, q_{H}^{\prime}\right)\right)(h)=T^{A} \subseteq\left\{i_{k}, i_{l}, \ldots, i_{n}\right\}$ where $\left|T^{A}\right| \leq\left|\left\{i_{k}, i_{l}, \ldots, i_{r}\right\}\right|$. Thus, $\left(q_{h}, q_{-h}^{\prime}\right) \succeq_{h}^{\vec{\varphi}} q_{H}^{\prime}$ by the responsiveness of hospitals' preferences.

The intuition underlying the above result is that any hospital that sets its regular market quota to its total capacity admits all of the interns achievable under the unique stable matching system right in the regular market. Moreover, since the matchings are determined by the serial dictatorship of interns, by reducing its regular market quota no hospital can ever hire any 'desirable yet unachievable' intern, who would not choose this hospital (under the report of the total capacity) while it was available in his or her choice

\footnotetext{
${ }^{10}$ Theorem 2 readily extends to a matching environment where interns have common preferences and consequently the unique stable matchings are obtained by the serial dictatorship of hospitals.
} 
set. In fact, any hospital reducing its regular market quota may lose some of the interns that it could otherwise employ (under the report of the total capacity) to some of the other hospitals.

\section{Conclusions}

In this paper, we studied a capacity allocation game played by hospitals in a hospital-intern market with an aftermarket. We showed that a purestrategy equilibrium may not exist under the intern-optimal stable matching system. We also established that common preferences for hospitals ensure the existence of equilibrium, where setting the regular market quota to the total capacity becomes a weakly dominant strategy for each hospital. But, strong monotonicity of population is not a sufficient restriction on preferences to avoid the nonexistence problem, like in games of capacity manipulation. In games of capacity allocation, it is not true either that every hospital weakly prefers a mixed-strategy Nash equilibrium to any larger regular market quota profiles.

Hospital-intern markets, as the motivating example of many-to-one matching in our model, have disallowed the direct use of intertemporal capacity allocation in the USA. Nevertheless, quota reversions used in the NRMP, in effect, create an indirect possibility of strategic allocation of capacity by hospitals. In the NRMP mechanism, each hospital program announces its ranking of the interns and its quota along with its reversion status. Quota reversions allow programs to donate unfilled positions to other programs during the matching process. Although it has been argued in the NRMP User Guide (2007) that institutions can use reversions to provide some degree of protection against the possibility that positions will go unfilled, ${ }^{11}$ reversions may easily facilitate strategic manipulation of capacity allocation. For instance, a program may announce a low quota for a popular program and a high quota for an unpopular one with a reversion option to the popular one.

\footnotetext{
${ }^{11}$ The NRMP User Guide (2007) indicates that reversion is primarily used between categorical and preliminary programs, between internal medicine and internal medicine primary care, or between tracks.
} 
By restricting the quota of the popular program a priori, the hospital may ensure a match with a better set of interns.

In the design of the existing NRMP algorithm, computational experiments aiming to test the algorithm's vulnerability to the capacity manipulation à la Sonmez (1999) and its sensitivity to the sequence in which quota reversions are processed were conducted and later reported in Roth and Peranson (1999). In these sensitivity tests, the input program quotas were set to be the final postmatch quotas produced by preexisting NRMP algorithm (one in use before 1997) and newly proposed intern-optimal stable matching algorithm. The results of the experiments showed that only negligible number of applicants and programs were affected by altering the time when reversions enter the match process. However, these experimental tests take the number of positions (of programs) to be reverted to be fixed by data, and therefore they do not target to check for the scope of manipulation of outcomes through quota reversions, i.e. the problem of capacity allocation in hospital-intern markets.

A better-known example of capacity allocation games is the early admission game played by many colleges in the USA for the last fifty years. ${ }^{12}$ Our findings in this paper uncovered the instrumental role played by early admission programs in increasing the bargaining power of colleges (vis à vis students) by making the supply side of the market 'thinner'. ${ }^{13}$ We believe that further research may profitably study intertemporal capacity allocation in college admission where preferences of colleges and students also enter as strategic variables into the play.

\section{References}

Abdulkadirog̃lu, A. and Sönmez, T. "Random Serial Dictatorship and the Core from Random Endowments in House Allocation Problems," Econometrica, 1998, 66, 689-701.

\footnotetext{
${ }^{12}$ See Avery, et al. (2003).

${ }^{13}$ The regular market and the aftermarket in the capacity allocation game we have studied can be considered to correspond to early admission and regular admission in the college admission problem.
} 
Avery, C., Fairbanks, A. and Zeckhauser, R. The Early Admission Game: Joining the Elite. Cambridge: Harvard University Press, 2003.

Gale, D. and Shapley, L.S. "College Admissions and the Stability of Marriage," American Mathematical Monthly, 1962, 69(1), 9-15.

Kojima, F. "Mixed Strategies in Games of Capacity Manipulation in HospitalIntern Markets," Social Choice and Welfare, 2006, 27(1), 25-28.

Konishi, H. and Ünver, M.U. "Games of Capacity Manipulation in HospitalIntern Markets," Social Choice and Welfare, 2006, 27(1), 3-24.

Nash, J.F. "Noncooperative Games," Annals of Mathematics, 1951, 54, 286295.

NRMP User Guide, 2007. Available at www.nrmp.org.

Papai, S. "Strategy-Proof Assignment by Hierarchical Exchange," Econometrica, 2000, 68, 1403-1433.

Roth, A.E. "The College Admissions Problem is not Equivalent to the Marriage Problem," Journal of Economic Theory, 1985, 36(2), 277-288.

Roth, A.E. and Peranson, E. "The Redesign of Matching Markets for American Physicians: Some Engineering Aspects of Economic Design," American Economic Review, 1999, 89, 748-780.

Roth, A.E. and Sotomayor, M. Two-Sided Matching: A Study in Game Theoretic Modeling and Analysis. London/New York: Cambridge University Press, 1990.

Sönmez, T. "Manipulation via Capacities in Two-Sided Matching Markets," Journal of Economic Theory, 1997, 77(1), 197-204.

Sönmez, T. "Can Pre-Arranged Matches Be Avoided in Two-Sided Matching Markets?," Journal of Economic Theory, 1999, 86(1), 148-156.

Svensson, L.G. "Queue Allocation of Indivisible Goods," Social Choice and Welfare, 1994, 11, 323-330. 